\title{
LOS RECURSOS Y LAS CAPACIDADES DE LA POLITICA EXTERIOR DE MÉXICO (2012-2018)
}

\author{
THE RESOURCES AND CAPABILITIES OF MEXICAN \\ FOREIGN POLICY
}

(2012-2018)

\section{LES RESSOURCES ET LES CAPACITÉS DE LA POLITIQUE ÉTRANGÈRE DU MEXIQUE}

(2012-2018)

\author{
Jorge A. Schiavon \\ Centro de Investigación y Docencia Económicas (CIDE) \\ jorge.schiavon@cide.edu \\ Bruno Figueroa Fischer \\ Servicio Exterior Mexicano \\ bfigueroa@sre.gob.mx
}

Resumen: Este artículo analiza los recursos y las capacidades de la política exterior de México durante la administración del presidente Enrique Peña Nieto (2012-2018), en particular, el presupuesto aprobado y ejercido por la Secretaría de Relaciones Exteriores, SRE, así como su tasa de crecimiento anual y los recursos destinados a temas estratégicos. También estudia los poderes de coordinación de la SRE y la dispersión de la acción internacional de México. Asimismo, analiza la evolución del personal diplomático y del Servicio Exterior Mexicano y, finalmente, examina las capacidades de política exterior en dos áreas fundamentales: la atención consular y los recursos dedicados a la cooperación internacional, la promoción comercial, cultural y de imagen. El artículo concluye que hubo una importante desinversión de recursos y capacidades en política exterior durante esta administración: el presupuesto se redujo 13\% en términos reales en dólares y bajó como porcentaje del presupuesto federal total; la coordinación de la acción internacional de otras secretarías y entidades públicas por parte de la cancillería fue muy limitada; la presencia de México en el mundo se mantuvo relativamente constante y no refleja la impor- 
tancia del país a nivel global; hubo una significativa disminución del personal administrativo en la cancillería; los recursos consulares fueron limitados y, en gran medida, provistos de modo excepcional por otras secretarías; finalmente, la cooperación internacional, la promoción y la diplomacia pública contaron con magros recursos para su ejecución en la segunda mitad del gobierno.

Palabras clave: recursos; política exterior; México; Enrique Peña Nieto; atención consular; cooperación internacional; promoción comercial.

ABSTRACT: This article analyzes the resources and capabilities of Mexico's foreign policy during the administration of President Enrique Peña Nieto (20122018), in particular, the budget approved and exercised by the Ministry of Foreign Affairs (SRE), and its annual growth rate and the resources allocated to strategic issues. It also studies the powers of coordination of the SRE and the distribution of Mexico's actions on the international stage as well as the evolution of diplomatic personnel and of the Mexican Foreign Service and, finally, examines foreign policy capabilities in two fundamental areas: consular assistance and resources devoted to international cooperation, and commercial, cultural and image promotion. The article concludes that there was a significant disinvestment of foreign policy resources and capacities during this administration: the budget fell by $13 \%$ in real dollar terms and decreased as a percentage of the total federal budget; coordination with the international actions of other ministries and public entities by the foreign ministry was very limited; Mexico's presence in the world remained relatively unchanged and failed to reflect the importance of the country at the global level; there was a significant decrease in administrative personnel in the foreign ministry; consular resources were limited and, to a large extent, provided exceptionally by other ministries; finally, international cooperation, promotion and public diplomacy were only provided with limited resources for implementation in the second half of the government.

Keywords: resources; foreign policy; Mexico; Enrique Peña Nieto; consular assistance; international cooperation; commercial promotion.

\section{Traducción de Gonzalo Celorio Morayta}

Résumé: L'article analyse les ressources et les capacités de la politique étrangère du Mexique sous la présidence d'Enrique Peña Nieto, en particulier en ce qui concerne le budget approuvé et le budget réellement dépensé par le Mi- 
nistère des Relations extérieures (SRE): on cite les taux de croissance annuelle, surtout pour ce qui est des ressources destinées à des objectifs stratégiques. D'autres sujets abordés sont le travail de coordination du SRE, la dispersion des activités internationales du Mexique, et l'évolution du personnel diplomatique et du Service extérieur mexicain. On parle aussi des capacités de la politique étrangère dans deux domaines fondamentaux. Le premier est celui des services consulaires, tandis que le second correspond aux efforts pour la coopération internationale, ainsi que pour la promotion du commerce, de la culture et de l'image du pays. L'article signale un déclin important des moyens et des capacités de la politique étrangère sous le gouvernement en question: le budget a souffert d'une réduction de 13\% en termes réels (c'est à dire en dollars), et sa part dans les dépenses totales du gouvernement fédéral a baissé de même. Le rôle du SRE en tant que coordinateur des activités internationales d'autres ministères et organismes publics s'est affaibli. La présence du Mexique sur la scène internationale, quoique constante, n'a guère correspondu à l'importance du pays. Le personnel administratif du SRE a diminué sensiblement. Les ressources destinées aux consulats, fort restreintes, maintes fois ont été procurées à titre exceptionnel par d'autres ministères. La coopération internationale, les travaux de promotion et la diplomatie publique n'ont disposé que d'une recette limitée, surtout dans la seconde moitié de la période présidentielle qui nous occupe.

Mots clefs: Ressources; politique étrangère; Mexique; Enrique Peña Nieto; services consulaires; coopération internationale; promotion du commerce.

Traducción de Bernardo Mabire

Fecha de recepción: diciembre de 2018

Fecha de aceptación: febrero de 2019 
L A Secretaría de Relaciones Exteriores (SRE o cancillería) es la institución gubernamental responsable de coordinar y ejecutar la política exterior en México. ${ }^{1}$ Como toda institución pública, es necesario que cuente con las capacidades legales, presupuestales, de infraestructura y personal para llevar a cabo sus funciones de manera óptima. El objetivo central de este artículo es estudiar los recursos y capacidades de los que dispone la SRE para cumplir sus funciones. Para hacerlo, como lo establece la escasa bibliografía sobre análisis de política exterior de los ministerios de relaciones exteriores (MRE),$^{2}$ se analizarán algunos de los principales indicadores para medir sus capacidades institucionales, como los poderes legales (centralización de facultades), recursos financieros (presupuesto y su distribución), infraestructura diplomática (número y tamaño de representaciones en el exterior), recursos humanos (personal y su profesionalización), así como el uso de recursos en dos áreas centrales, la actividad consular y la cooperación internacional.

${ }^{1}$ Ley Orgánica de la Administración Pública Federal, México, 1976 (última reforma, 30 de noviembre de 2018), http://www.diputados.gob. $\mathrm{mx} /$ LeyesBiblio/pdf/153_301118.pdf

${ }^{2}$ Brian Hocking, "Catalytic Diplomacy: Beyond 'Newness' and 'Decline'”, en Jan Melissen, (ed.), Innovation in Diplomatic Practice, Londres, Palgrave Macmillan, 1999, pp. 19-42; Christian Lequesne, Ethnographie du Quai d'Orsay. Les pratiques des diplomates français, París, CNR Editions, 2017; Neumann, Iver B., At Home with Diplomats: Inside a European Foreign Ministry, Ithaca, Cornell University Press, 2012; David Clarck, "Neoliberalism and Public Service Reform: Canada in Comparative Perspective”, Canadian Journal of Political Science, 35 (2002), pp. 771-793; Andrew F. Cooper y Timothy Shaw, The Diplomacies of Small States: Between Vulnerability and Resilience, Londres, Palgrave Macmillian, 2009; Andrew F. Cooper, Brian Hocking y William Maley, Global Governance and Diplomacy: Worlds Apart?, Nueva York, Palgrave Macmillian, 2008; Jérémie Cornut y Vincent Pouliot, "Practice Theory and the Study of Diplomacy: A Research Agenda", Cooperation and Conflict 50 (2015), pp. 297-315; Kishan S. Rana, The Contemporary Embassy: Paths to Excellence. Nueva York, Palgrave Macmillan, 2013; y Jeffrey Robertson, Diplomatic Style and Foreign Policy: A Case Study of South Korea, Nueva York, Routledge, 2016. 
Según Brian Hocking, todos los gobiernos nacionales desarrollan un conjunto de instrumentos para interactuar con el mundo y ejecutar su política internacional. Esto es conocido como el sistema diplomático nacional (SDN), el cual reúne un conjunto de instituciones encargadas de relaciones con el exterior. Los MRE generalmente son los responsables de coordinar la definición y ejecución de la política exterior y hay una tendencia a equipararlos al SDN. Sin embargo, no son las únicas instituciones involucradas en las relaciones externas de un país. En algunos casos, los MRE enfrentan rivalidades con otras instancias gubernamentales y tienen responsabilidades internacionales. ${ }^{3}$

Aun cuando el SDN puede estar integrado por distintas instituciones, los MRE y sus representaciones diplomáticas en el exterior son la pieza fundamental en la conducción de la política exterior. Son muy limitados los estudios que analizan los recursos y capacidades diplomáticas de los MRE a nivel internacional ${ }^{4}$ y en particular el caso de la SRE mexicana. ${ }^{5}$ Un factor descuidado de manera recurrente por los

${ }^{3}$ Brian Hocking, "The Ministry of Foreign Affairs and the National Diplomatic System", en Pauline Kerr y Geoffrey Wiseman (eds.), Diplomacy in a Globalizing World: Theories and Practices, Nueva York, Oxford University Press, 2013, pp. 123-140.

${ }^{4}$ Valentin Naumescu, "Diplomatic Services Today: Between Political Decisions and Administrative Criteria", Transylvanian Review of Administrative Sciences, 44 (2015), pp.161-179; Yiwel Wang, "Public Diplomacy and the Rise of Chinese Soft Power". The Annals of the American Academy of Political and Social Science, 616 (2008), pp. 257-273, y Kishan S. Rana, The Contemporary Embassy..., op. cit.

${ }^{5}$ Rafael Velázquez Flores y Gabriel Farfán Mares, "Presupuesto y política exterior en México: planeación estratégica, incrementalismo y discrecionalidad", Revista Legislativa de Estudios Sociales y de Opinión Pública, 5 (2012), pp. 39-101; Bruno Figueroa y Jorge A. Schiavon, "Brasil y México: Inversión y capacidades en política exterior", Foreign Policy Edición Mexicana, 3 (2014), pp. 12-15; Jorge A. Schiavon y Bruno Figueroa. "Las capacidades de la política exterior de México: diagnóstico (2000-18) y propuestas (2018-24)", en Jorge A. Schiavon, Rafael Velázquez Flores y Humberto Garza Elizondo (eds.), La política exterior de México 2018-2024: diagnóstico y propuestas, México, CIDE, UABC y UANL, 2018, pp. 17-24. 
analistas internacionales es el de los recursos con los que cuentan las diplomacias de cada país para llevar a cabo sus funciones. Esto no es un tema menor, como lo subrayó Oliver Stuenkel: "mientras los poderes emergentes buscan proyectar más influencia, el reducido número de sus diplomáticos impone severas limitaciones a su capacidad de ejecutar nuevas políticas". 6

Para México, el factor internacional es de gran trascendencia en su desarrollo nacional y para el bienestar de los mexicanos. Con las reformas económicas iniciadas en la década de 1980 y el cambio de modelo de desarrollo hacia la promoción de la inversión extranjera directa y las exportaciones, el comercio internacional del país (importaciones más exportaciones) representa actualmente (2017) más de tres cuartas partes del PIB $(77.57 \%)$, es decir, uno de los principales motores económicos nacionales. Junto con el turismo, proporciona trabajo de manera directa e indirecta a millones de mexicanos. Según datos del Banco Mundial, ese mismo año los ingresos internacionales del país alcanzaron máximos históricos: inversión extranjera directa (31.7 mil millones de dólares), remesas (30.6 mil millones de dólares) e ingresos turísticos (20.6 mil millones de dólares), lo que representa en conjunto $7.21 \%$ del PIB. ${ }^{7}$ Asimismo, la presencia de más de 12 millones de connacionales fuera del territorio, equivalente a cerca del $10 \%$ de la población total, obliga a un enorme esfuerzo de atención por parte del gobierno.

Siendo así, se esperaría que México contara con una infraestructura de política exterior acorde con su apertura internacional y que reflejara la relevancia del factor externo en la seguridad y el desarrollo nacionales, así como en el bienestar de los mexicanos. Para saber con qué recursos y

${ }^{6}$ Oliver Stuenkel, "How many diplomats does an emerging power need?", Post-Western World (blog personal), 14 de octubre 2012, http:// www.postwesternworld.com/2012/10/14/how-many-diplomats-does-anemerging-power-need/

${ }^{7}$ Banco Mundial, World Development Indicators, 2018, http://datatopics.worldbank.org/world-development-indicators/ 
capacidades cuenta México en general y la SRE en particular, este artículo analizará a profundidad los indicadores básicos en cuanto al presupuesto y la organización de la SRE durante la administración del presidente Enrique Peña Nieto (20122018), en particular, los datos correspondientes al presupuesto aprobado y ejercido, la tasa de crecimiento anual y los recursos destinados a temas estratégicos; también se analizará el número de personal diplomático (total, per cápita y por representación), así como su tasa de crecimiento anual; asimismo, se estudiará la presencia en el exterior (número de representaciones total y per cápita, así como su distribución a nivel mundial, por región y por bloque).

El artículo se divide en seis secciones. La primera analiza el presupuesto de la SRE; la segunda estudia los poderes de coordinación de la SRE y la dispersión de la acción internacional de México; la tercera examina la red diplomática y consular; la cuarta analiza la evolución del personal del Servicio Exterior Mexicano (SEM); la quinta estudia el presupuesto para la atención consular y por último, la sexta examina los recursos dedicados a la cooperación internacional, la promoción comercial, cultural y de imagen, varias de las cuales se conocen como "diplomacia pública". ${ }^{8}$ Las conclusiones resumen los principales hallazgos y proponen algunas acciones de aplicación inmediata que podrían mejorar los recursos y capacidades de la política exterior de México.

\section{EL PRESUPUESTO PARA POLÍTICA EXTERIOR}

¿Cómo ha evolucionado el presupuesto de la SRE en las últimas dos décadas y, en particular, durante la administración del presidente Peña Nieto? Como puede apreciarse el cuadro 1, en pesos corrientes, el presupuesto ejecutado de la

8 César Villanueva Rivas, "Construyendo el poder suave de México", Revista Mexicana de Política Exterior, 111 (2017). 
SRE ha aumentado de 3.416 mil millones de pesos ( $\mathrm{mmp}$ ) en 2000 a 5.819 mmp en 2006, a 9.784 mmp en 2012 y a 13.065 mmp en 2017. Esto significa que, en términos nominales, el presupuesto aumentó $282.47 \%$ en los últimos tres sexenios: $70.36 \%$ con Vicente Fox (2000-2006), 68.14\% con Felipe Calderón (2006-2012), y menos de la mitad que en las dos administraciones anteriores $(33.53 \%)$ con Peña Nieto.

\section{CuAdro 1}

Presupuesto ejecutado por la SRE (2000-2017)

\begin{tabular}{ccccc}
\hline Año & $\begin{array}{c}\text { Miles de pesos } \\
\text { corrientes }\end{array}$ & $\begin{array}{c}\text { Miles de USD } \\
\text { corrientes }\end{array}$ & $\begin{array}{c}\text { Miles de USD } \\
\text { constantes }(2000)\end{array}$ & $\begin{array}{c}\text { Porcentaje del } \\
\text { presupuesto federal }\end{array}$ \\
\hline 2000 & $\$ 3415821.90$ & $\$ 361249.84$ & $\$ 361249.84$ & $0.332 \%$ \\
2001 & $\$ 3707389.60$ & $\$ 396832.34$ & $\$ 386023.68$ & $0.348 \%$ \\
2002 & $\$ 3845119.80$ & $\$ 398212.14$ & $\$ 381265.64$ & $0.328 \%$ \\
2003 & $\$ 4572976.50$ & $\$ 423854.80$ & $\$ 396693.10$ & $0.351 \%$ \\
2004 & $\$ 5350391.50$ & $\$ 474074.72$ & $\$ 432029.99$ & $0.374 \%$ \\
2005 & $\$ 5215459.20$ & $\$ 478575.06$ & $\$ 421790.34$ & $0.321 \%$ \\
2006 & $\$ 5819219.00$ & $\$ 533910.45$ & $\$ 455968.96$ & $0.307 \%$ \\
2007 & $\$ 7096998.01$ & $\$ 649421.08$ & $\$ 538986.48$ & $0.351 \%$ \\
2008 & $\$ 7483581.00$ & $\$ 672396.36$ & $\$ 537625.04$ & $0.290 \%$ \\
2009 & $\$ 7688538.80$ & $\$ 568953.49$ & $\$ 456742.65$ & $0.335 \%$ \\
2010 & $\$ 8997652.70$ & $\$ 712064.48$ & $\$ 562626.80$ & $0.364 \%$ \\
2011 & $\$ 8696824.40$ & $\$ 700040.00$ & $\$ 535974.65$ & $0.320 \%$ \\
2012 & $\$ 9784370.40$ & $\$ 742959.21$ & $\$ 557135.23$ & $0.338 \%$ \\
2013 & $\$ 8200865.80$ & $\$ 642097.65$ & $\$ 474384.71$ & $0.262 \%$ \\
2014 & $\$ 9306476.00$ & $\$ 700132.48$ & $\$ 509115.27$ & $0.272 \%$ \\
2015 & $\$ 10866812.40$ & $\$ 685422.72$ & $\$ 497920.86$ & $0.289 \%$ \\
2016 & $\$ 13497822.90$ & $\$ 723483.90$ & $\$ 518825.42$ & $0.320 \%$ \\
2017 & $\$ 13064595.91$ & $\$ 690185.38$ & $\$ 484766.27$ & $0.313 \%$ \\
\hline
\end{tabular}

Fuente: elaboración propia con información de la Cuenta Pública de la SHCP, http:/ / cuentapublica. hacienda.gob.mx/ transformados a USD y deflactados con datos del Banco Mundial, http://datatopics.worldbank. org/world-development-indicators/ 
Sin embargo, cerca del $70 \%$ del presupuesto de la SRE es ejecutado en divisas extranjeras. Si se calcula el presupuesto en dólares corrientes al tipo de cambio promedio del año, éste pasó de 361.250 millones de dólares (md) en 2000, a 533.910 en 2006, a 742.959 en 2012, a 690.185 en 2017. Siendo así, se observa un incremento acumulado en las tres últimas administraciones de $91.05 \%$, aumentado $47.80 \%$ con Fox, $39.15 \%$ con Calderón, pero que cayó $-7.10 \%$ con Peña Nieto. Ahora bien, tomando en cuenta la inflación promedio anual en Estados Unidos para deflactar los presupuestos y hacerlos comparables en términos reales, el presupuesto ejecutado por la cancillería pasó de 361.250 millones de dólares constantes del año 2000 (md2000), a 421.790 en 2006, a 557.135 en 2012, y a 484.766 en 2017. Esto implica que, en términos constantes en dólares, el presupuesto de la SRE aumentó en $34.19 \%$ desde el año 2000, creciendo $26.22 \%$ con Fox, $22.19 \%$ con Calderón y decreciendo $-12.99 \%$ con Peña Nieto, lo cual significa que, en términos reales en dólares, en la administración del presidente Peña Nieto la capacidad de gasto de la cancillería se redujo prácticamente $13 \%$.

Para conocer la importancia de la política exterior dentro de las políticas públicas nacionales, un buen indicador en cuanto al gasto es el porcentaje del presupuesto federal total que representa el presupuesto ejecutado por la sRE. Como puede observarse en el cuadro 1, en 2000, éste representaba el $0.332 \%$ del presupuesto total, pasando a $0.307 \%$ en 2006 , $0.338 \%$ en 2012 y, finalmente, $0.313 \%$ en 2017 . Lo anterior significa que el presupuesto de la cancillería, como porcentaje del presupuesto federal total, cayó $-5.51 \%$ en el periodo analizado, bajando $-7.42 \%$ con Fox, subiendo $10.06 \%$ con Calderón y cayendo $-7.26 \%$ con Peña Nieto.

Si en política las prioridades se reflejaran en el presupuesto, los datos anteriores demostrarían que la política exterior no ha sido una prioridad nacional en el presente siglo, particularmente en la administración del presidente Peña Nieto. La presencia internacional y la importancia de lo externo han aumentado para México en los últimos años, pero los recur- 
sos financieros destinados a la política externa van a la baja en términos absolutos y relativos, en dólares constantes del 2000, en la SRE y como porcentaje del presupuesto federal.

\section{LA COORDINACIÓN DE LA POLÍTICA EXTERIOR}

Aunque la SRE tiene la facultad de coordinar la política exterior de México, muchas secretarías de Estado y organismos públicos llevan a cabo acciones internacionales, la mayoría de las veces poco coordinadas entre ellas. Por ejemplo, durante el gobierno del presidente Peña Nieto, ProMéxico se encargó de la promoción de exportaciones y atracción de inversiones extranjeras, el Consejo de Promoción Turística de México (СРTM) promovió al país como destino turístico, mientras que la Secretaría de Cultura (sc) (antes Consejo Nacional para la Cultura y las Artes, Conaculta) llevó a cabo una intensa promoción de la cultura nacional a nivel global y la Presidencia de la República coordinó la imagen internacional y la "marca país".

Para conocer la relevancia de la política exterior frente a otras políticas públicas, es necesario comparar el presupuesto ejecutado por la SRE con otras secretarías, para identificar si las variaciones presupuestales son similares o diferentes entre éstas. Como ya se apuntó, en términos reales en dólares, entre 2000 y 2017 el presupuesto ejecutado por la SRE se incrementó $34.19 \%$. En el mismo periodo, los presupuestos de otras secretarías y organismos con incidencia internacional aumentaron en los siguientes montos: SC, 391.81\%; CPT, $224.56 \%$ y ProMéxico, $-7.81 \%$. Como puede observarse, salvo en el caso de ProMéxico, el incremento al presupuesto de la SRE es sustantivamente menor que la SC y el CPT. En particular, durante el sexenio de Peña Nieto los datos siguen la misma tendencia: SRE, -12.99\%; sc, 34.17\%; CPT, $11.94 \%$ y ProMéxico, $-37.89 \%$ (véase la gráfica 1). Como puede observarse, el gran perdedor en lo que respecta a presupuesto es ProMéxico, pero la sRe también pierde casi $13 \%$ de su presu- 
puesto mientras que, aun en momentos de austeridad y recortes presupuestales como los de la segunda mitad del sexenio, la SC y el CPT tienen ganancias presupuestales.

\section{GRÁFICA 1}

Presupuesto ejecutado por dependencias federales seleccionadas en México (2000-2017) (miles de dólares constantes de 2000)

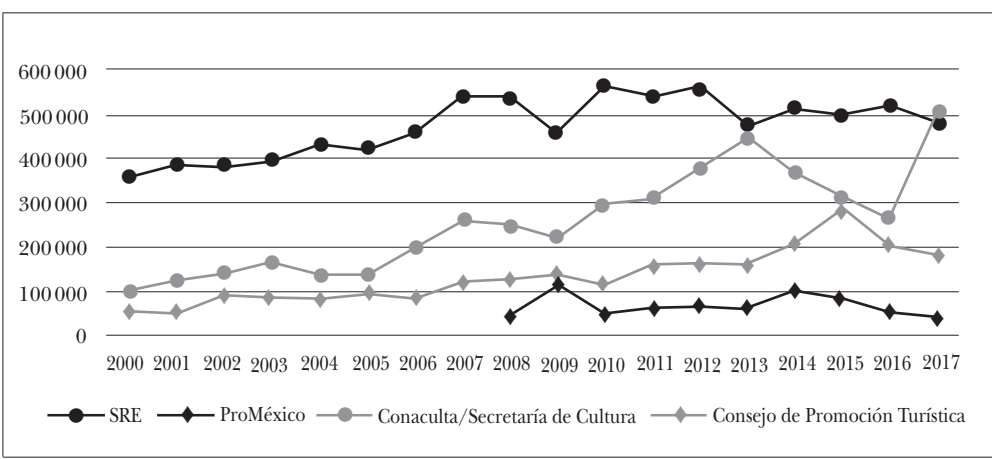

Fuente: elaboración propia con información de la Cuenta Pública de la SHCP, http://cuentapublica. hacienda.gob.mx/ transformados a USD y deflactados con datos del Banco Mundial, http://datatopics.worldbank. org/world-development-indicators/

Además, durante la administración Peña Nieto no existió un mecanismo intersecretarial integral a través del cual la cancillería pudiera coordinar las acciones de todas estas dependencias y organismos. Así, salvo ProMéxico, la SRE no sólo se vio más afectada que otras instancias en términos presupuestales, sino que también careció de los mecanismos y recursos para coordinar las acciones internacionales de éstas. ${ }^{9}$

${ }^{9}$ El presidente Andrés Manuel López Obrador (2018-2024) decidió cerrar las oficinas de promoción económica (ProMéxico) y turística (СРTM) de México en el exterior. Estas responsabilidades fueron transferidas a la 


\section{LA RED DIPLOMÁTICA Y CONSULAR MEXICANA}

Hay una estrecha relación entre el tamaño de las economías del mundo y la dimensión de sus redes diplomáticas. De acuerdo con Global Diplomacy Index (GDI), elaborado por el Lowy Institute de Australia, ${ }^{10}$ las diez redes diplomáticas más grandes del mundo (sólo embajadas), corresponden a nueve de las diez principales economías globales. Solamente Canadá (décima economía) se encuentra fuera de esta lista, en el lugar 15, mientras que Rusia, la economía número 11 del mundo de acuerdo con el Banco Mundial, por PIB nominal, se encuentra en cuarta posición, al haber heredado las embajadas de la Unión Soviética. Por su parte, México tiene 80 embajadas (2018) y ocupa el lugar 28 (empatando con Grecia), muy lejos de su lugar 15 entre las economías del mundo. Sólo en América Latina, Brasil (economía número nueve del mundo) cuenta con la novena red diplomática (137 embajadas), 57 más que México, mientras que Argentina (vigésimo primera economía) tiene cinco embajadas más. ${ }^{11}$

En términos de distribución regional, México concentra la mayoría de sus embajadas en las Américas (26) y Europa (24). África es el continente donde su presencia es más débil, con ocho embajadas con las que debe cubrir 54 países. En Medio Oriente, Asia y Oceanía se encuentra presente sólo en los 20 países económicamente más importantes. ${ }^{12}$ Así, México sólo tiene embajadas en $41.45 \%$ de los 194 países del mundo (incluyendo al Estado Vaticano).

SRE, sin que, al mes de enero 2019, se le hayan otorgado recursos financieros o humanos adicionales para realizarlas.

${ }^{10}$ Lowy Institute, "Global Diplomacy Index", https:/ / globaldiploma cyindex.lowyinstitute.org

11 Jorge A. Schiavon y Bruno Figueroa. "La paradoja mexicana: Un país abierto al mundo con limitadas capacidades diplomáticas". Foreign Affairs Latinoamérica, 19, 1, (2019), en prensa.

${ }^{12}$ SRE, "Consulados de México en el Exterior", gob.mx (sitio de internet), https:/ / directorio.sre.gob.mx/index.php/consulados-de-mexicoen-el-exterior 
Sin embargo, México cuenta con una de las redes consulares más amplias del mundo, con 67 consulados generales y de carrera. ${ }^{13}$ Es el país del G20 con la proporción más elevada de consulados frente al total de representaciones diplomáticas (incluye embajadas, consulados, misiones ante organismos internacionales y otras oficinas como Taiwán). En su gran mayoría se concentran en Estados Unidos y Canadá, con 50 y cinco consulados respectivamente. Así, sumando el número total de representaciones diplomáticas (156), México ocupa el lugar 18 en el mundo, de acuerdo con el CDI de Lowy, muy cerca de su nivel económico global (15). ${ }^{14}$

La administración Peña Nieto incrementó moderadamente la red diplomática mexicana con la apertura de cinco nuevas embajadas, dos consulados de carrera y la reapertura de la misión ante la UNESCo. Sin embargo, para acercarse al número de embajadas que tienen otras economías emergentes como México, necesitaría incrementar su red diplomática a un ritmo promedio de cinco nuevas al año y alcanzar así a Sudáfrica en seis años, a la India en diez, a Turquía en 11, a Brasil en 12 y a China en 16.

En el sexenio que concluyó, también se amplió la red de oficinas de promoción comercial de ProMéxico, de 31 oficinas en 21 países (2012) a 48 oficinas (2018). Sin embargo, este número es considerablemente menor a las 126 representaciones comerciales de Corea del Sur (коTRA) o las 84 de Australia (Austrade). Por su parte, para la promoción turística, el CPTM contaba con 21 oficinas en 13 países (2018), mismo número que en 2012, y sólo cuatro más que en 2006. Otra vez, este número es sustantivamente menor que en países de tamaño similar a México, como Turquía (dos lugares abajo en términos de turistas recibidos), con 45 (2018) oficinas de cultura y turismo en el extranjero. ${ }^{15}$ art. cit.

13 Loc. cit.

14 Jorge A. Schiavon y Bruno Figueroa. "La paradoja mexicana...",

15 Loc. cit. 


\section{El Servicio Exterior Mexicano}

La Secretaría de Relaciones Exteriores es la única dependencia federal en la que convive personal regido por distintas disposiciones legales y administrativas, nacionales y extranjeras. En México, dispone de personal que labora al amparo de la Ley Federal de los Trabajadores al Servicio del Estado, ${ }^{16}$ así como los miembros del Servicio Exterior Mexicano (SEM), que cuentan con su propia normatividad, la Ley del Servicio Exterior Mexicano (LSEM). ${ }^{17}$ En las representaciones del exterior, además de los miembros del SEM, trabaja personal local regido por las leyes laborales de cada país.

Este conjunto de trabajadores compone los recursos humanos de la cancillería, dedicados a la ejecución de la política exterior. Su estudio estaría incompleto si sólo se enfocara en una categoría, como podría ser el propio SEM, tan esencial como el personal local en las embajadas y consulados o el personal que labora en México.

El Informe de rendición de cuentas de la Administración Pública Federal, 2006-2012, presentado al final del gobierno de Calderón, hizo un recuento detallado de los recursos humanos de la cancillería por categoría de plazas. ${ }^{18}$ En el cuadro 2 se presenta la situación de esas plazas, al 30 de noviembre de 2012 y al 30 de noviembre de 2018, de acuerdo con las cifras que figuran en el Informe de rendición de cuentas correspondiente al gobierno de Peña Nieto. ${ }^{19}$ La única información ausente es la del personal local en el exterior.

${ }^{16}$ Ley Federal de los Trabajadores al Servicio del Estado, México, 28 de diciembre de 1963 (última reforma, 22 de junio de 2018), http:// www.diputados.gob.mx/LeyesBiblio/pdf/111_220618.pdf.

17 Ley del Servicio Exterior Mexicano, México, 4 de enero de 1994 (última reforma, 19 de abril de 2018) http://www.diputados.gob.mx/ LeyesBiblio/pdf/96_190418.pdf

18 SRE, "Informe de rendición de cuentas de la Administración Pública Federal 2006-2012”, México, https://sre.gob.mx/images/stories/doc transparencia/transparencia/rc/ic2006-2012.pdf

19 SRE, "Informe de rendición de cuentas de conclusión de la administración 2012-2018”, México, https://www.gob.mx 


\section{CUADro 2}

Recursos humanos de la SRE por categoría de plazas

\begin{tabular}{ccccc}
\hline Tipo de plaza & $\begin{array}{c}\text { 30 de enero } \\
\text { de } 2012\end{array}$ & $\begin{array}{c}\text { 3 de noviembre } \\
\text { de } 2018\end{array}$ & Diferencia & $\%$ \\
\hline Estructura & 2135 & 1915 & -220 & $-10.31 \%$ \\
$\begin{array}{c}\text { Contratos por honorarios } \\
\text { Eventuales }\end{array}$ & 105 & 35 & -70 & $-66.67 \%$ \\
$\begin{array}{c}\text { Eventuales financiadas por } \\
\text { plazas de estructura }\end{array}$ & 993 & 610 & -383 & $-38.57 \%$ \\
$\quad$ Subtotal sin sEM & 3381 & 560 & -148 & $-100 \%$ \\
$\begin{array}{c}\text { Plazas del sem autorizadas } \\
\text { en el Presupuesto de Egresos }\end{array}$ & & & & $-24.28 \%$ \\
de la Federación (PEF) & 1430 & 1487 & +57 & $+3.99 \%$ \\
$\quad$ Total con SEM & 4811 & 4047 & -764 & $-15.88 \%$ \\
\hline
\end{tabular}

Fuente: SRE, Informes de rendición de cuentas 2006-2012 y 20122018.

Se pueden derivar varias conclusiones del cuadro 2. Primero, con respecto a la plantilla laboral, excluyendo al SEM, durante la administración Peña Nieto la SRE perdió 821 trabajadores y funcionarios. El informe de labores de esta administración da cuenta, año con año, de manera sistemática, de la aplicación de distintas medidas de austeridad y disciplina fiscal que generaron la reducción del personal; estas reducciones son, por categoría: estructura $(-10.31 \%)$; contratos por honorarios $(-66.67 \%)$, eventuales $(-38.57 \%)$ y eventuales financiadas por plazas de estructura $(-100 \%) .{ }^{20}$ En conjunto, esta reducción equivale a $24.28 \%$ del personal que no forma parte del SEM. Lo anterior tiene un impacto importante en las capacidades para realizar las funciones administrativas, ya que hay áreas donde no es fácil incrementar la productividad. La informática puede ayudar a compensar la pérdida de una secretaria, pero la cancelación de un puesto de responsabilidad obliga a que otro funcionario asuma las tareas

${ }^{20}$ El Quinto Informe de Labores de la SRE (2016-2017) refiere, por ejemplo, la cancelación de 79 plazas, p. 251. 
de quien se ha ido, incrementando su carga de trabajo sustancialmente.

La Secretaría de Hacienda y Crédito Público (shcP) accedió, en cambio, a un incremento de 57 plazas para el SEM, pasando de 1430 a 1487, entre 2012 y 2018, lo cual representa un aumento de $3.99 \%$. Estas plazas las ocupan tanto los miembros del SEM de carrera, como algunos que no lo son. ${ }^{21}$ Los miembros del SEM de carrera pueden trabajar en México con su propia plaza, por lo que muchas veces estas plazas del SEM sirven para llenar los huecos dejados por una reducción de otro tipo de plazas.

A lo largo del presente siglo, el personal del SEM de carrera ha ido disminuyendo paulatinamente: de 1367 (diciembre de 2001), bajó a 1129 (diciembre de 2006) y disminuyó ligeramente a 1123 (diciembre de 2012) cuando asumió la presidencia Peña Nieto. La última cifra publicada (junio de 2018) es de 1128 miembros, cinco más que al inicio de la administración. ${ }^{22}$ En relación con su población, México tiene hoy aproximadamente un diplomático profesional por cada 100000 habitantes; en comparación, en 1975 había uno por cada 50000 . Es paradójico que hoy México tenga, relativo a su población, $50 \%$ menos diplomáticos de carrera que los que tenía hace 40 años.

Cabe preguntarse si, dada la importancia del plano internacional para México, aunado a la amplia red de representaciones en el exterior y de delegaciones en el país, así como a las necesidades de la estructura central de la propia cancillería: ¿es suficiente el número de miembros del SEM en 2018 para realizar las tareas que requiere la política exterior de la décimoquinta economía del mundo? Todo indica que las necesidades son mayores a las capacidades existentes. Al térmi-

${ }^{21}$ SRE, Sexto Informe de Labores 2018, Anexo estadístico "Relaciones bilaterales y multilaterales". Para noviembre de 2018, 145 plazas hasta el rango de ministro las ocupaba personal de designación política, conocido como "por artículo 7" de la LSEM.

${ }^{22}$ Son diferencias marginales debido a las renuncias, fallecimientos o jubilaciones en el momento del recuento. 
no del gobierno de Felipe Calderón, la canciller Patricia Espinosa dejó como legado una memoria documental sobre la evaluación y el desarrollo del sem de carrera. En ésta, solicitó a la SHCP considerar la creación de 150 nuevas plazas. ${ }^{23}$

Dado que el incremento en el número de miembros del SEM no ha llegado a la cifra de 150, la preocupación externada en esta memoria documental sigue siendo de actualidad: en promedio, hay menos personal de carrera por representación que hace seis años: 7.2 frente a 7.7.24 Ha sido necesario incluso crear una nueva categoría dentro de la rama técnico-administrativa para los expertos en sistemas que dan servicio a la red diplomática y consular en el exterior, como "personal informático regional”, que sumaban 40 a finales de 2018.

El presente análisis de los recursos humanos de la cancillería debe igualmente incorporar una perspectiva cualitativa, y hacer referencia a las acciones llevadas a cabo para apoyar el desarrollo profesional de los miembros de carrera del SEM, principalmente por el Instituto Matías Romero (IMR), así como a la importante reforma a la LSEM y su reglamento efectuada en 2018. Pocas dependencias de gobierno tienen la oferta de formación continua como lo hace la SRE para sus funcionarios, tanto presencial como a distancia. Entre enero 2013 y junio de 2018, el IMR ofreció cursos de idiomas y de materias relativas a la vasta gama de habilidades requeridas en la carrera diplomática, muchos con valor curricular, desde negociación internacional hasta documentación consular. El IMR capacitó a los miembros del SEM, ofreciendo 3659 matrículas en cursos presenciales y a distancia (299 ediciones de cursos a distancia y 137 cursos presenciales); además,

23 SRE, "Evaluación y desarrollo del Servicio Exterior Mexicano de Carrera (2006-2012): Memoria documental", México, https://sre.gob.mx/ images/stories/doctransparencia/rdc/memodoc/12mddgserhsem.pdf

${ }^{24}$ El resultado se obtiene dividiendo el número de miembros del SEM en el exterior (personal diplomático-consular y técnico-administrativo) entre el número de representaciones, en 2011 y en 2018, de acuerdo con los Informes de Labores e Informes de rendición de cuentas respectivos. 
se ofrecieron 2098 matrículas en estos cursos a servidores públicos de la SRE que no eran miembros del SEM. ${ }^{25}$ Más importante aún, el IMR introdujo un nuevo enfoque en su labor formativa, poniendo mayor énfasis en la capacitación de habilidades y actitudes, por ejemplo, en el tema de habilidades gerenciales y de liderazgo, o en materia de equidad de género, frente a la tradicional consolidación de conocimientos.

La reforma a la LSEM aprobada en abril de 2018, así como su Reglamento, expedido en noviembre del mismo año, son de gran relevancia para mejorar las condiciones laborales del personal de carrera del SEM. Estas reformas fueron calificadas por uno de los diplomáticos de mayor rango y experiencia como "las de mayor calado en la última generación”, ${ }^{26}$ y buscaron fortalecer las tres etapas de la carrera de los diplomáticos: ingreso, desarrollo profesional y jubilación. ${ }^{27}$ Además, introducen el concepto de "Plan de carrera" como herramienta para ofrecer certidumbre en la planeación de carreras individuales, tomando en cuenta, a la vez, las necesidades de la Secretaría. La nueva Ley fortalece, entre otros, la equidad de género, el ascenso meritocrático, permite laborar hasta los 70 años y asegura condiciones de retiro más dignas.

En suma, ante un panorama de amplia reducción de recursos humanos en la cancillería en el gobierno de Peña Nieto -mayor que en las dos administraciones anterioreslas notas positivas fueron el incremento en la formación profesional y la profunda reforma a la LSEM, que mejora las perspectivas de desarrollo porfesional y de vida de los diplomáticos mexicanos de carrera.

25 IMR-SRe, "Informe de rendición de cuentas de conclusión de la administración 2012-2018, https:/ /www.gob.mx/

${ }^{26}$ Carlos de Icaza, "Discurso pronunciado durante la ceremonia de promulgación de la Lsem", Ciudad de México, 18 de abril de 2018 (discurso compartido por el autor).

${ }^{27}$ Decreto por el que se reforman, adicionan y derogan diversas disposiciones de la Ley del Servicio Exterior Mexicano, Diario Oficial de la Federación, 19 de abril de 2018. 


\section{LOS RECURSOS CONSULARES}

La labor consular de México es titánica, al atender a una comunidad de más de 12 millones de mexicanos en el exterior, $93 \%$ de ellos en Estados Unidos. En ese país hay 11.6 millones de personas nacidas en México, más 23 millones de descendientes de mexicanos de segunda y subsecuentes generaciones (aproximadamente la mitad de segunda y la otra mitad de subsecuentes generaciones). Se estima que 5.8 millones de ellos se encuentran en una situación migratoria irregular ${ }^{28}$ o tienen un estatus legal temporal o precario (DACA, DAPA, H2A). De los 5.8 millones que gozan de una situación migratoria regular, se calcula que 3.2 millones son residentes permanentes legales y el resto tiene visas temporales. ${ }^{29}$ Sin importar su situación migratoria, todos ellos pueden requerir servicios consulares.

Para cumplir con las múltiples tareas que realizan los consulados en distintos ámbitos (documentación, protección, atención comunitaria, así como acciones de promoción, imagen y relacionamiento político a nivel local), la cancillería ha puesto en marcha varias medidas para paliar las limitaciones de recursos financieros y humanos. La primera ha sido asegurar un "piso" de recursos destinados a la red consular, en particular para documentación y protección, a través de los ingresos consulares, como lo marca el artículo 2, fracción XI, de la LSEM en su reforma de 2002. ${ }^{30}$

28 A. González Barrera y J. M. Krogstad, "What we know about illegal immigration from Mexico", Pew Research Center, 2016, http://www. pewresearch.org/fact-tank/2017/03/02/what-we-know-about-illegal-immigration-from-mexico/

${ }^{29}$ James Lee and Bryan Baker, "Estimates of the Lawful Permanent Resident Population in the United States: January 2014", Estados Unidos, Department of Homeland Security, junio de 2017, https:/ /www.dhs.gov/ sites/default/files/publications/LPR\% 20Population \% 20Estimates\%20 January\% 202014.pdf

${ }^{30} \mathrm{El}$ artículo 2, fracción XI, dice: "Destinar los ingresos recibidos por los servicios establecidos en la Ley Federal de Derechos, prestados por cualquier representación consular en el extranjero, con el objeto de cu- 
La cuenta consular, es decir, los recursos que se obtienen en los consulados por la provisión de servicios, son canalizados a la Tesorería de la Federación, que los reintegra a la SRE para su aplicación en los propios consulados. La intención original de los autores de esta reforma era asegurar recursos complementarios a los otorgados a la red consular vía el presupuesto ordinario. Con el paso de los años, los ingresos consulares se han convertido en la fuente preponderante de financiamiento de esta labor para cubrir los gastos de operación y mantenimiento de los consulados; cuando resultan insuficientes, se coomplementan con recursos cada vez más reducidos del presupuesto ordinario de la SRE. Programas de protección como el Programa de Asistencia Jurídica a Mexicanos a través de Asesorías Legales Externas en Estados Unidos (PALE) o el Programa de Asistencia Jurídica a Casos de Pena Capital en Estados Unidos (MCLAP), han tenido que financiarse con recursos del presupuesto ordinario.

La principal desventaja de este esquema es la complejidad de la administración presupuestal que supone y la merma sustancial de recursos por su manejo financiero. En los movimientos de ida y vuelta de los ingresos consulares desde y hacia Estados Unidos, con dos cambios de moneda y un porcentaje que queda en la Secretaría de Hacienda y Crédito Público (9\% en 2018), la pérdida neta para la cancillería en lo que respecta a estos ingresos es superior al 20\%. ${ }^{31}$ Además, no es posible conocer de antemano la cifra de recaudación final, lo cual genera una fuerte incertidumbre al preparar el presupuesto del año siguiente.

Otro paliativo a las limitaciones presupuestarias ha sido la canalización de recursos extraordinarios provenientes de otras dependencias o instancias a la labor consular, incluyen-

brir, previa autorización de la Secretaría, los gastos relativos a las actividades y programas de la misma, en términos del Reglamento”.

31 Información proporcionada a los autores en noviembre de 2018 por un alto funcionario de la SRE. 
do adecuaciones por parte de la SHCP. Durante el gobierno del presidente Peña Nieto, destacan en particular una transferencia para protección consular del Instituto Nacional Electoral (INE) en 2017, y diversas transferencias al Instituto de los Mexicanos en el Exterior (IME) para el apoyo educativo, entre ellos IME becas por parte de la Secretaría de Educación Pública (SEP) y para el programa de ventanillas de salud, financiado por la Secretaría de Salud (ss).

En 2017, el INE acordó renunciar a un proyecto inmobiliario y poner a disposición de las autoridades hacendarias los 1070 millones de pesos reservados para ese fin, para la ejecución de "actividades prioritarias". ${ }^{32}$ Se decidió usar dichos recursos para labores consulares con la llegada de Donald Trump a la presidencia en Estados Unidos. En febrero del mismo año, la SHCP acordó con la SRE la calendarización de la entrega de los recursos y su distribución en cinco rubros para cada uno de los 50 consulados en Estados Unidos. ${ }^{33}$ Éstos constituyeron el programa conocido como "Fortalecimiento de la Atención de los Mexicanos en Estados Unidos (FAMEU)". En 2018, los 300 millones de pesos no gastados el año anterior se canalizaron para los mismos fines a través de un nuevo contrato. Debe notarse que parte de este monto se utilizó para el sostenimiento de la red consular, ante las limitaciones presupuestales señaladas. Cabe destacar que estos recursos sólo se recibieron en una ocasión y no se regularizaron en el presupuesto de 2018.

32 Zedryk Raziel, "Cancela INE sede; reintegra mil mdp", en Reforma, 12 de enero de 2017.

33 SRE, "Recursos extraordinarios para la protección de mexicanos en Estados Unidos de América" (comunicado de prensa), 28 de febrero de 2017, https://www.gob.mx; y SRE, "Recursos extraordinarios para la protección de mexicanos en Estados Unidos de América”, 28 de febrero de 2017 https://www.gob.mx Estos rubros fueron: 1) Recursos humanos; 2) Programa de Protección a Mexicanos; 3) Programa de Servicios Consulares; 4) Ventanillas de asesoría financiera y protección al patrimonio; y 5) Apoyo a migrantes a través de las Delegaciones. 
El IME, por su parte, recibió ampliaciones presupuestarias de parte de la SHCP para el desempeño de sus labores. El cuadro 3 revela las fluctuaciones en el presupuesto del IME entre 2014 y julio de 2018.

\section{Cuadro 3}

IME: presupuesto original, modificado y ejercido

(31 de diciembre del año, miles de pesos)

\begin{tabular}{cccccc}
\hline Año & Original & Modificado & Ejercido & $\begin{array}{c}\text { Diferencia } \\
\text { ejercido-original }\end{array}$ & $\begin{array}{c}\text { \% de incremento entre } \\
\text { original y ejercido }\end{array}$ \\
\hline 2014 & $\$ 33321$ & $\$ 201944$ & $\$ 201876$ & $\$ 168555$ & $505.85 \%$ \\
2015 & $\$ 69393$ & $\$ 501591$ & $\$ 561572$ & $\$ 492179$ & $709.26 \%$ \\
2016 & $\$ 59068$ & $\$ 963864$ & $\$ 955269$ & $\$ 896201$ & $1517.23 \%$ \\
2017 & $\$ 61475$ & $\$ 159600$ & $\$ 134980$ & $\$ 73505$ & $119.57 \%$ \\
20181 & $\$ 56036$ & $\$ 90502$ & $\$ 46938$ & n.a. & n.a. \\
Suma & $\$ 279293$ & $\$ 1917501$ & $\$ 1900635$ & $\$ 1621342$ & $580.51 \%$ \\
\hline
\end{tabular}

Fuente: elaboración propia con información de la Cuenta Pública de la SHCP, http://cuentapublica. hacienda.gob.mx/

${ }^{1}$ al 31 de julio de 2018; n.a. = no aplica.

Con base en el cuadro 3, pueden sacarse varias conclusiones. El presupuesto original asignado al IME creció $68.17 \%$ entre 2014 y 2018, pasando de 33.321 a 56.036 millones de pesos. Sin embargo, el presupuesto ejercido fue muy superior al original, tomando en cuenta la magnitud de las ampliaciones presupuestarias: en 2014, la SHCP transfirió y el IME ejerció recursos 505\% superiores al presupuesto inicial; en 2015 fueron $709 \%$ mayores y, en 2015, $1517 \%$ superiores. En 2017, las ampliaciones se redujeron, para ser sólo $119 \%$ mayores al presupuesto original. En cuatro años y medio, los recursos transferidos por la SHCP al IME sumaron casi $2 \mathrm{mmp}$ (\$1 917501000), monto de gran importancia en comparación con los modestos recursos originales asignados al IME desde el presupuesto de la cancillería (279.293 millones de pesos) en el mismo periodo. Gran parte de los recursos fue- 
ron canalizados a asociaciones estadounidenses como EM-USA Foundation y Parents Alliance Inc. para la entrega de becas escolares. ${ }^{34}$ Cabe subrayar que en estos años se ejercieron montos extraordinarios a través del IME, los cuales tan pronto como llegaron a la SRE, fueron transferidos a otras asociaciones. Así, el presupuesto original en 2018 representa $5.86 \%$ de lo que se ejerció sólo dos años atrás, en 2016. Oscilaciones presupuestales de estas magnitudes sin duda tienen importantes efectos en la planeación y ejecución de cualquier programa, en este caso, la atención a comunidades en el exterior.

Otro programa que ha tercerizado el IME a través de asociaciones sin fines de lucro en Estados Unidos, han sido las Ventanillas de Salud (vDs), programa interinstitucional entre la Secretaría de Salud (ss) y la SRE, mediante el cual la primera transfiere, cada año desde 2015, 40 millones de pesos a la segunda. Cada consulado mexicano en Estados Unidos cuenta con su ventanilla, manejada por una o varias de las 600 agencias socias locales. ${ }^{35}$ La Dra. Gudelia Martínez, Secretaria Ejecutiva de la Sección Mexicana de la Comisión de Salud Fronteriza México-Estados Unidos, realizó una estimación de costos de los principales servicios brindados durante 2017, con base en la población beneficiada, calculando un monto de 143 mdd; a éste deben sumarse salarios y otros aportes monetarios y no monetarios para el fortalecimientos de las VDs, incluyendo los modestos aportes de los consulados, como uso de espacios y electricidad, por poco más de

${ }^{34}$ Estas asociaciones sin fines de lucro estaban vinculadas a la organización Juntos Podemos de la excandidata presidencial Josefina Vázquez Mota. Este esquema y su poca transparencia suscitó en los medios y en la sociedad mexicana numerosos cuestionamientos cuyo análisis rebasa el presente artículo.

${ }^{35}$ Ver Secretaría de Salud y SRE, "Estrategias de Ventanillas de Salud; informe ejecutivo", (s/f) documento interno, y sRE, "Lineamientos de las Ventanillas de Salud (vDs)", documento en línea, https://sre.gob.mx/ component $/$ phocadownload $/$ category $/ 7$-normateca-interna?download= 639:lineamientos-de-las-ventanillas-de-salud-vds 
medio millón de dólares. ${ }^{36}$ Por lo tanto, "la aportación del gobierno de México para la Estrategia Ventanillas de Salud es considerada como un recurso semilla, ya que las aportaciones de las agencias que operan las vDs y de aliados estratégicos representa un $91.3 \%$ del monto estimado que se requiere para brindar servicios preventivos básicos." ${ }^{37} \mathrm{En}$ suma, en el caso de la atención en salud a la comunidad, y visto desde la perspectiva de los recursos invertidos por el gobierno de México y el valor de los servicios otorgados, el modelo parece haber sido extremadamente exitoso.

Finalmente, es importante analizar el uso intensivo de recursos humanos en el ámbito consular. Según Hernández Joseph, en 2014, el 52\% del personal del sem fuera del país tenía funciones consulares, al igual que el $60 \%$ de los empleados locales, ${ }^{38}$ cuando los consulados sólo representan el $45 \%$ del total de representaciones mexicanas en el exterior. Aun ante la considerable inversión de capital humano en temas relativos al consulado, éstos siguen siendo insuficientes para cubrir todas las acciones y programas consulares. Por ello, habría que sumar a todas las personas que, a través de esquemas de colaboración, apoyan en diversidad de actividades, de la protección (abogados pro bono) al acercamiento con la comunidad (organizaciones locales en la gestión de las becas ImE y de las Ventanillas de Salud). Ante la dimensión cotidiana del trabajo consular, el reto es no sólo mejorar los indicadores cuantitativos, sino también mejorar la calidad y ampliar o actualizar la gama de los servicios que se ofrecen a la comunidad. ${ }^{39}$

${ }^{36}$ Gudelia Rangel, "Estimación de costos de los servicios que se brindan a través de la Estrategia Ventanillas de Salud”, sept. 2018, documento entregado a los autores, p. 3.

37 "Estrategias de Ventanillas de Salud", doc. cit., p. 21.

38 Hernández Joseph, Protección consular mexicana, México, Miguel Ángel Porrúa y Fundación Ford, 2016, p. 238.

${ }^{39}$ Una buena reflexión sobre la insuficiencia de la atención cualitativa en el ámbito de la protección se encuentra en el capítulo 6 de la obra 


\section{Los recursos de la Agencia Mexicana de Cooperación INTERNACIONAL PARA EL DESARROLLO}

Al establecerse por ley la Agencia Mexicana de Cooperación Internacional para el Desarrollo (Amexcid), en septiembre de 2011, se decidió que heredaría las atribuciones de la Unidad de Relaciones Económicas y Cooperación Internacional (Ureci). Durante el gobierno del presidente Peña Nieto sus responsabilidades siguieron siendo las mismas: cooperación internacional (educativa y cultural, científico y técnica, financiera), asistencia humanitaria, y promoción comercial, cultural y turística, coordinándose en su caso con otras instituciones públicas competentes. ${ }^{40}$ Tampoco quedó modificada la disposición transitoria de dicha Ley sobre su fuente de financiamiento, por la que todas las tareas de cooperación internacional debían sujetarse a la disponibilidad presupuestaria del PEF. ${ }^{41}$ Los recursos de la Agencia han provenido del propio presupuesto de la SRE, y han padecido por igual sus fluctuaciones, como puede apreciarse en el cuadro 4.

\section{Cuadro 4}

Amexcid: Presupuesto ejercido (millones de pesos)

\begin{tabular}{ccccccc}
\hline Año & 2013 & 2014 & 2015 & 2016 & 2017 & 2018 \\
\hline Monto & 419.0 & 373.8 & 414.3 & 619.1 & 363.4 & $447.0^{\mathrm{a}}$ \\
\hline
\end{tabular}

Fuente: Elaboración propia con base en información de Amexcid y Cuenta Pública, diversos años.

a Presupuesto modificado.

referida de Hernández Joseph, "Los retos para determinar métricas de desempeño y racionalización del uso de los recursos", op. cit.

${ }^{40}$ Ley de Cooperación Internacional para el Desarrollo, México, 6 de abril de 2011 (última reforma, 17 de diciembre de 2015), http://www. diputados.gob.mx/LeyesBiblio/pdf/LCID_171215.pdf

41 Artículo 2 transitorio de misma ley. 
Después de llegar a un pico en 2016 de 619 millones de pesos, $47.7 \%$ superior a la cifra de 2013 , el presupuesto de la agencia cayó en 2017 por debajo inclusive del monto del primer año de gobierno, para estabilizarse en 2018.

La contracción presupuestal y las prioridades de la cancillería afectaron severamente el presupuesto de la Amexcid a partir de 2016. Dicho por su director ejecutivo,

una vez descontados los gastos de operación, las cuotas a organismos internacionales y gastos por traslado, el margen de acción presupuestaria es en promedio $10 \%$ del presupuesto asignado originalmente, lo que obliga a la Amexcid a innovar en su interacción con los diferentes actores de la cooperación mexicana [cabe recordar que la Amexcid no es ejecutora de proyectos], por ejemplo haciendo deducibles de impuestos las aportaciones al Fondo Nacional de Cooperación Internacional para el Desarrollo (Foncid). ${ }^{42}$

Como ilustración de las oscilaciones presupuestales de la SRE y Amexcid en ámbitos concretos, se presenta en el cuadro 5 la evolución de la ayuda humanitaria mexicana gestionada por la Amexcid con recursos de la SRE y, a partir de septiembre de 2016, parcialmente con recursos de la Amexcid depositados en un "Fondo de Ayuda Humanitaria”, dentro del Fondo de Cooperación Internacional para el Desarrollo (Foncid). Este fondo canalizó, entre septiembre de 2016 y junio de 2018, 3.7 millones de pesos de ayuda, equivalentes a sólo 195000 dólares. Cabe notar que entre 2014 y 2015 la ayuda humanitaria mexicana cayó $95 \%$.

No debe pasarse por alto, sin embargo, que la cooperación internacional de México no es canalizada exclusivamente por la Amexcid, por lo que las acciones en la materia no dependen exclusivamente de las fluctuaciones presupuesta-

${ }^{42}$ Agustín García-López Loaeza, "La cooperación mexicana para el desarrollo: el poder suave de la política exterior", Revista Mexicana de Politica Exterior, 111, (2017), p. 32. 
les de la cancillería y en particular de la Agencia. El cuadro 6 muestra que, a diferencia del presupuesto de la Amexcid, la cooperación en 2017 fue la más importante del sexenio, descontando la cifra de $2013 .{ }^{43}$

\section{CuAdro 5}

Ayuda humanitaria canalizada a través de la Amexcid (dólares)

\begin{tabular}{cccccc}
\hline Año & 2013 & 2014 & 2015 & 2016 & 2017 \\
\hline Monto & 1000000 & 6850000 & 363342 & 433346 & 1216546 \\
\hline
\end{tabular}

Fuente: "Ayuda humanitaria y prevención de desastres", México, Amexcid, https://www.gob.mx/amexcid/acciones-y-programas/ayuda-hu manitaria-y-prevencion-de-desastres-29331

\section{Cuadro 6}

Recursos de la cooperación internacional para el desarrollo de México, 2013-2017 (millones de dólares)

\begin{tabular}{cccccc}
\hline Año & 2013 & 2014 & 2015 & 2016 & 2017 \\
\hline Monto & 547.73 & 288.65 & 207.06 & 287.91 & 317.60 \\
\hline
\end{tabular}

Fuente: Juan Pablo Prado Lallande y Noel González Segura, "México y el PABA+40: promoviendo la gobernanza de la Cooperación Sur-Sur", Revista Española de Desarrollo y Cooperación, 43 (2018), p. 104; Información de 2017 proporcionada por Amexcid a los autores.

En síntesis, al igual que en el ámbito consular, la Amexcid ha tenido la capacidad de apalancar sus recursos decrecientes con los de otras dependencias públicas y diversos socios, bajo múltiples esquemas, que le han permitido dar continui-

${ }^{43}$ La elevada cifra de 2013, relativa a los años siguientes, se debe a la contabilización de la condonación del $70 \%$ de la deuda del gobierno de Cuba con Bancomext. El volumen más importante de la CID proviene de contribuciones de la SHCP a organismos internacionales dedicados al desarrollo. 
dad a los programas de cooperación y promoción que coordina con fuertes fluctuaciones entre un año y otro.

\section{Conclusiones}

Un estudio enfocado únicamente en los recursos de una entidad pública está incompleto mientras éstos no se vinculen con los objetivos planeados, las acciones ejecutadas y los resultados obtenidos. Sin embargo, esto sobrepasa el objetivo del presente artículo. No obstante, este novedoso enfoque de análisis de los recursos y capacidades de la política exterior mexicana pone de manifiesto las limitaciones o deficiencias estructurales con las que la SRE debe llevar a cabo sus funciones, lo cual tiene un claro impacto en los resultados de su gestión, identificado por años por los especialistas en la materia.

Durante el sexenio del presidente Peña Nieto, como se analizó en este artículo, aun ante las mejoras legales de la LSEM, hubo una importante desinversión en materia de recursos y capacidades para la política exterior: el presupuesto se redujo $13 \%$ en términos reales en dólares y bajó como porcentaje del presupuesto federal total; la coordinación de la acción internacional de otras secretarías y entidades públicas de la cancillería fue muy limitada; la presencia de México en el mundo se mantuvo relativamente constante, sin reflejar la importancia del país a nivel global; hubo una disminución del personal administrativo en la cancillería; los recursos consulares fueron limitados y, en gran medida, provistos de modo excepcional por otras secretarías. Finalmente, la cooperación internacional, la promoción y la diplomacia pública contaron con recursos muy magros para su ejecución.

Para un país inserto, como lo está México, en el mercado global, la política exterior debe servir como un instrumento fundamental para impulsar el desarrollo económico, político y social del país. Por ello, debe ser una política prioritaria en la estrategia de desarrollo nacional. Además, ante una co- 
yuntura internacional compleja como la vivida durante la administración de Peña Nieto, particularmente tras la salida del Reino Unido de la Unión Europea y la elección de Donald Trump en Estados Unidos, es necesario fortalecer la política exterior de México, para que sus acciones tengan mayor impacto. Por ello, los recursos asignados a la política exterior deben tratarse como una inversión, no un gasto, para promover el desarrollo nacional y el bienestar de todos los mexicanos.

Del análisis anterior se deriva que, para reconstruir los recursos y capacidades de la política exterior de México con el propósito de promover el desarrollo nacional, podrían ponerse en marcha algunas de las siguientes iniciativas: 1) aumentar sustantivamente los recursos presupuestales de la SRE; 2) fortalecer los poderes de coordinación de la SRE en las acciones internacionales de otras dependencias, para desplegar una política exterior integral, coherente y sólida a nivel global; 3) incrementar el número de miembros del sEM y en plazas administrativas; 4) ampliar la cobertura diplomática de México en el mundo, incrementando el número de embajadas para tener el nivel de otros países similares y diversificando geográficamente su ubicación; 5) fortalecer la red consular en Estados Unidos con mayores presupuestos y certidumbre en su ejercicio; 6) crear áreas especializadas en temáticas de alta relevancia internacional en la cancillería (como energía, comercio, medio ambiente, etc.) para coordinar mejor las acciones de otras secretarías en el ámbito mundial; 7) que la Cámara de Diputados asigne un presupuesto para la Amexid, su fondo de cooperación (Foncid), así como recapitalizar el Fondo de Yucatán; 8) crear un mecanismo para compensar las variaciones cambiarias del peso frente a las divisas extranjeras en el presupuesto; 9) reconstituir los puentes con otras secretarías, para lo cual podría recrearse el Gabinete de Política Exterior, presidido por la SRE; finalmente, 10) elaborar un diagnóstico objetivo, independiente y transparente sobre las necesidades en cuanto a re- 
cursos humanos, financieros y materiales de la cancillería, con recomendaciones concretas de acción.

\section{Bibliografía}

Banco Mundial, World Development Indicators (base de datos en línea), 2018, http://datatopics.worldbank.org/world-develop ment-indicators/ (consultado el 15 de noviembre de 2018).

Clarck, David, "Neoliberalism and Public Service Reform: Canada in Comparative Perspective", Canadian Journal of Political Science 35 (2002), pp. 771-793.

CoOper, Andrew F. y Timothy SHaw, The Diplomacies of Small States: Between Vulnerability and Resilience, Londres, Palgrave Macmillan, 2009.

Cooper, Andrew F., Brian Hocking y William Maley, Global Governance and Diplomacy: Worlds Apart?, Nueva York, Palgrave Macmillan, 2008.

Cornut, Jérémie y Vincent Pouliot, "Practice Theory and the Study of Diplomacy: A Research Agenda", Cooperation and Conflict 50 (2015), pp. 297-315.

Figueroa, Bruno y Jorge A. Schiavon, "Brasil y México: Inversión y capacidades en política exterior", Foreign Policy Edición Mexicana, 3 (2014), pp. -15.

García-López loaeza, Agustín, "La cooperación mexicana para el desarrollo: el poder suave de la política exterior", Revista Mexicana de Política Exterior, 111, (2017), p. 32.

González Barrera, A. y J. M. Krogstad, 2016, Pew Research Center, "What we know about illegal immigration from Mexico", http:/ /www.pewresearch.org/fact-tank/2017/03/02/whatwe-know-about-illegal-immigration-from-mexico/ (consultado el 15 de noviembre de 2018).

Hernández Joseph, Daniel, Protección consular mexicana, México, Miguel Ángel Porrúa y Fundación Ford, 2016.

Hocking, Brian, "Catalytic Diplomacy: Beyond 'Newness' and 'Decline'”, en Jan Melissen (ed.), Innovation in Diplomatic Practice, Londres, Palgrave Macmillan, 1999, pp. 19-42. 
Hocking, Brian, "The Ministry of Foreign Affairs and the National Diplomatic System", en Pauline Kerr y Geoffrey Wiseman (eds.), Diplomacy in a Globalizing World: Theories and Practices, Nueva York, Oxford University Press, 2013.

IcAzA, Carlos de, "Discurso pronunciado durante la ceremonia de promulgación de la LSEM", Ciudad de México, 18 de abril de 2018, s.p.i.

LEE, Sook Jong (ed.), Transforming Global Governance with Middle Power Diplomacy. South Korea's Role in the 21st Century, Nueva York, Palgrave Macmillan, 2016.

LeE, James y Bryan BAKer, "Estimates of the Lawful Permanent Resident Population in the United States: January 2014", Estados Unidos, Department of Homeland Security, 2014, https:// www.dhs.gov/sites/default/files/publications/LPR\%20Population\%20Estimates\%20January\%202014.pdf (consultado el 15 de noviembre de 2018).

Lequesne, Christian, Ethnographie du Quai d'Orsay. Les pratiques des diplomates français, París, CNR Editions, 2017.

Lowy Institute, Global Diplomacy Index (sitio de internet), 2018 https://globaldiplomacyindex.lowyinstitute.org (consultado el 15 de noviembre de 2018).

Naumescu, Valentin, "Diplomatic Services Today: Between Political Decisions and Administrative Criteria”, Transylvanian Review of Administrative Sciences, 44 (2015), pp. 161-179.

Neumann, Iver B., At Home with Diplomats: Inside a European Foreign Ministry, Ithaca, Cornell University Press, 2012.

Pamment, James, British Public Diplomacy and Soft Power: Diplomatic Influence and the Digital Revolution, Londres, Palgrave Macmillan, 2016.

Prado Lallande, Juan Pablo y Noel González Segura, "México y el PABA+40: promoviendo la gobernanza de la Cooperación Sur-Sur", Revista Española de Desarrollo y Cooperación, 43 (2018). RANGEL, Gudelia,"Estimación de costos de los servicios que se brindan a través de la Estrategia Ventanillas de Salud", septiembre de 2018, documento entregado a los autores por SRE, p. 3., s.p.i. RAzIEL, Zedryk, "Cancela INE sede; reintegra mil mdp", Reforma, 12 de enero de 2017. 
Rana, Kishan S., The Contemporary Embassy: Paths to Excellence, Nueva York, Palgrave Macmillan, 2013.

Robertson, Jeffrey, Diplomatic Style and Foreign Policy: A Case Study of South Korea, Nueva York, Routledge, 2016.

Schiavon, Jorge A. y Bruno FigueroA, "La paradoja mexicana: Un país abierto al mundo con limitadas capacidades diplomáticas”, Foreign Affairs Latinoamérica, 19, 1 (2019), en prensa.

Schiavon, Jorge A. y Bruno Figueroa, "Las capacidades de la política exterior de México: diagnóstico (2000-18) y propuestas (2018-24)", en Jorge A. Schiavon, Rafael Velázquez Flores y Humberto Garza Elizondo (eds.), La política exterior de México 2018-2024: Diagnóstico y Propuestas, México, CIDE, UABC y UANL, 2018, pp. 17-24.

Secretaría de Hacienda y Crédito Público, SHCP, Cuenta Pública de la shcP (base de datos en línea), http://cuentapublica.ha cienda.gob.mx/ (consultado el 15 de noviembre de 2018).

Secretaría de Relaciones Exteriores, SRE, "Recursos extraordinarios para la protección de mexicanos en Estados Unidos de América" (comunicado de prensa), México, 28 de febrero de 2017, https://www.gob.mx/sre/prensa/recursos-extraordinarios-pa ra-la-proteccion-de-mexicanos-en-estados-unidos-deamerica?idiom=es (consultado el 15 de noviembre de 2018).

Secretaría de Relaciones Exteriores, SRE, "Consulados de México en el Exterior" (sitio de internet), https://directorio.sre.gob. $\mathrm{mx} /$ index.php/consulados-de-mexico-en-el-exterior (consultado el 15 de noviembre de 2018).

Secretaría de Relaciones Exteriores, SRE, "Evaluación y desarrollo del Servicio Exterior Mexicano de Carrera (2006-2012): Memoria documental", 2012, https://sre.gob.mx/images/sto ries/doctransparencia/rdc/memodoc/12mddgserhsem.pdf (consultado el 15 de noviembre de 2018).

Secretaría de Relaciones Exteriores, SRE, "Lineamientos de las Ventanillas de Salud (vDs)”, https://sre.gob.mx/component/ phocadownload/category/7-normateca-interna?download $=6$ 39:lineamientos-de-las-ventanillas-de-salud-vds (consultado el 15 de noviembre de 2018). 
Secretaría de Relaciones Exteriores, SRE, "Recursos extraordinarios para la protección de mexicanos en Estados Unidos de América", 28 de febrero de 2017, https://www.gob.mx/cms/ uploads/attachment/file/195660/reursos_extraoridnarios_ para_proteccio_n_de_mexicanos.pdf (consultado el 15 de noviembre de 2018).

Stuenkel, Oliver, "How many diplomats does an emerging power need?" (blog del autor), 14 de octubre 2012, http:/ / www.post westernworld.com/2012/10/14/how-many-diplomats-does an-emerging-power-need/ (consultado el 15 de noviembre de 2018).

Velázquez Flores, Rafael y Gabriel Farfán Mares, "Presupuesto y política exterior en México: planeación estratégica, incrementalismo y discrecionalidad", Revista Legislativa de Estudios Sociales y de Opinión Pública, 5 (2012), pp. 39-101.

Villanueva Rivas, César, "Construyendo el poder suave de México”, Revista Mexicana de Política Exterior, 111 (2017).

Wang, Yiwel, "Public Diplomacy and the Rise of Chinese Soft Power". The Annals of the American Academy of Political and Social Science, 616 (2008), pp. 257-273.

\section{INFORMES, LEYES E INSTRUMENTOS DE DERECHO INTERNACIONAL}

"Decreto por el que se reforman, adicionan y derogan diversas disposiciones de la Ley del Servicio Exterior Mexicano”, Diario Oficial de la Federación, 19 de abril de 2018.

IMR-SRE, "Informe de rendición de cuentas de conclusión de la administración 2012-2018", México, 2018, https://www.gob.mx/ cms/uploads/attachment/file/405879/Informe_de_ Rendici_n_de_Cuentas_de_Conclusi_n_de_la_Administra ci_n_2012-2018_del_Instituto_Mat_as_Romero.pdf (consultado el 15 de noviembre de 2018).

Ley de Cooperación Internacional para el Desarrollo, México, 6 de abril de 2011 (última reforma, 17 de diciembre de 2015). 
Ley del Servicio Exterior Mexicano, México, 4 de enero de 1994 (última reforma, 19 de abril de 2018).

Ley Federal de los Trabajadores al Servicio del Estado, México, 28 de diciembre de 1963 (última reforma, 22 de junio de 2018).

Ley Orgánica de la Administración Pública Federal, México, 1976 (última reforma, 30 de noviembre de 2018).

Secretaría de Relaciones Exteriores, SRE, "Informe de rendición de cuentas de conclusión de la administración 2012-2018”, 2018, https://www.gob.mx/cms/uploads/attachment/file/410264/ Informe_de_Rendici_n_de_Cuentas_SRE_firmado_por_Can ciller.pdf (consultado el 15 de noviembre de 2018).

Secretaría de Relaciones Exteriores, sRE, "Informe de rendición de cuentas de la Administración Pública Federal 2006-2012”, 2012, https://sre.gob.mx/images/stories/doctransparencia/ transparencia/rc/ic2006-2012.pdf (consultado el 15 de noviembre de 2018).

Secretaría de Salud y Secretaría de Relaciones Exteriores, SRE, "Estrategias de Ventanillas de Salud; informe ejecutivo" (documento sin fecha entregado a los autores), s.p.i. 